Devel opment of el ectromagnet i $c$ and pi ezoel ectric hybrid act uat or system

\begin{tabular}{|c|c|}
\hline 著者 & $\begin{array}{l}\text { OKEYA Ryot a, AOYAG Nanabu, TAKANO } \\
\text { Takehi ro, TAMURA Hi deki }\end{array}$ \\
\hline $\begin{array}{l}\text { jour nal or } \\
\text { publ } \mathrm{i} \text { cat } \mathrm{i} \text { on } \mathrm{title}\end{array}$ & Sensor s and act uat or s. A, Physi cal \\
\hline vol une & 200 \\
\hline page $r$ ange & $155-161$ \\
\hline year & $2013-10-01$ \\
\hline URL & ht t p: //hdl . handl e. net /10258/2736 \\
\hline
\end{tabular}


Devel opment of el ectromagnet i $c$ and pi ezoel ectric hybrid act uat or system

\begin{tabular}{|c|c|}
\hline 著者 & $\begin{array}{l}\text { OKEYA Ryot a, AOYAG Nanabu, TAKANO } \\
\text { Takehi ro, TAMURA Hi deki }\end{array}$ \\
\hline $\begin{array}{l}\text { jour nal or } \\
\text { publ } \mathrm{i} \text { cat } \mathrm{i} \text { on } \mathrm{title}\end{array}$ & Sensor s and act uat or s. A, Physi cal \\
\hline vol une & 200 \\
\hline page $r$ ange & $155-161$ \\
\hline year & $2013-10-01$ \\
\hline URL & ht t p: //hdl . handl e. net /10258/2736 \\
\hline
\end{tabular}




\title{
Development of electromagnetic and piezoelectric hybrid actuator system
}

\author{
Ryota Okeya, Manabu Aoyagi, Takehiro Takano, Hideki Tamura
}

\begin{abstract}
An ordinal force-feedback device typically uses an electromagnetic motor (EMM), which provides an excellent expression of elasticity. However, it is not easy to express the sense of hardness and roughness because the response of the current is delayed due to the inductance of the armature winding. On the contrary, a piezoelectric actuator, which has a rapid response, is good at expressing the sense of hardness and roughness. Thus, if different types of actuators are used in the same actuator system (AS), the weaknesses of each type can be compensated for. In this study, as an ideal force-feedback device, a hybrid actuator system combining an EMM with an ultrasonic motor (USM) and a piezoelectric clutch/brake (piezo-clutch/brake) is proposed and examined. This AS can expand the range of representable feelings.

This paper describes the construction of a hybrid AS and some experimental results of a force-feedback display. In this experiment, the feelings of roughness, friction, and elasticity were represented. The feeling of roughness was represented by the on-off control of the piezo-brake at defined positions. The feeling of friction was represented by the PID control of braking using the piezo-clutch. The feeling of elasticity was represented by two methods: the use of the EMM and brake and the use of a combination of the USM, clutch, and brake. As a result, the hardness feeling was realistically represented by the piezo-brake, and the elastic feeling was represented by either the EMM or the USM.
\end{abstract}

Keyword: haptics interface; hybrid; piezo electric actuator 


\section{Introduction}

Force-feedback devices can reproduce the feeling of a virtual object displayed on a computer display. Such devices can be applied to a variety of fields including engineering design, surgical training, and entertainment [1-3]. The training of handling of medical devices such as dental and computer tomography (CT) have been reported [4-6]. Many of researched and developed force-feedback devices mainly use electromagnetic motors (EMMs) [7-9], and they are excellent at expressing the feeling of elasticity. However, it is not easy to express a realistic sense of hardness or roughness using such devices because the representation of hardness requires a high speed control of EMM [10,11]. In general, haptic control frequency required is more than $1 \mathrm{kHz}$ [12]. If the hardness feeling is represented at the control frequency less than $1 \mathrm{kHz}$, the user will get a sense like that even hard objects is dented. Hence an ideal actuator system for a haptic display has required to be developed, and the authors have studied it so far.

In previous our study, an actuator system using an ultrasonic motor (USM) and a piezoelectric clutch/brake (piezo-clutch/brake) has been proposed and evaluated [13,14]. A typical USM is driven by the friction force between a rotor and a stator under the condition that a preload is always applied between the stator and the rotor. A USM can not only generate thrust but also braking force using its self-holding force. Therefore, it is difficult to reproduce a torque-free state that allows the rotor to undergo free motion following the application of an external force. To solve this problem, a piezo-clutch was developed to separate the USM from the rotor. In general, it is hard to arbitrarily change the preload of an ordinal USM during operation. However, a USM with a piezo-clutch can electrically control the preload during operation. Moreover, the piezo-clutch is also useful for force feedback because this is faster than conventional clutches such as an electromagnetic clutch. The USM is effective to represent high-speed reaction force such as repulsive forces or collision. Such an actuator system (AS) is effective at expressing sensations of hardness and roughness but may be unsuitable for elastic sensations.

The objective of this study is to develop a new hybrid AS that includes an EMM and a USM. An AS using multilayer piezoelectric actuators (MPAs) has performance characteristics that are opposite to those of an AS using EMMs [2,13]. If different types of actuators are used in the same AS, the individual actuators can complement each other, allowing the weaknesses of each to be compensated for so that the range of representable feelings can be expanded. In this paper, the construction of the hybrid AS and some experimental results of a force-feedback display trial are presented.

\section{Construction and Operating Principles}

Figure 1(a) shows the trial construction of the proposed hybrid AS. It is composed of a USM and an EMM, which generate the thrust, and a piezo-clutch/brake based on a mechanical amplifier, which is used for preload and braking adjustments. A lever attached to a rotating shaft transmits the feeling to the user. The user can simply receive of the feelings of the virtual objects by the rotation of lever. A photograph of the prototype device is shown in Fig. 1(b). 


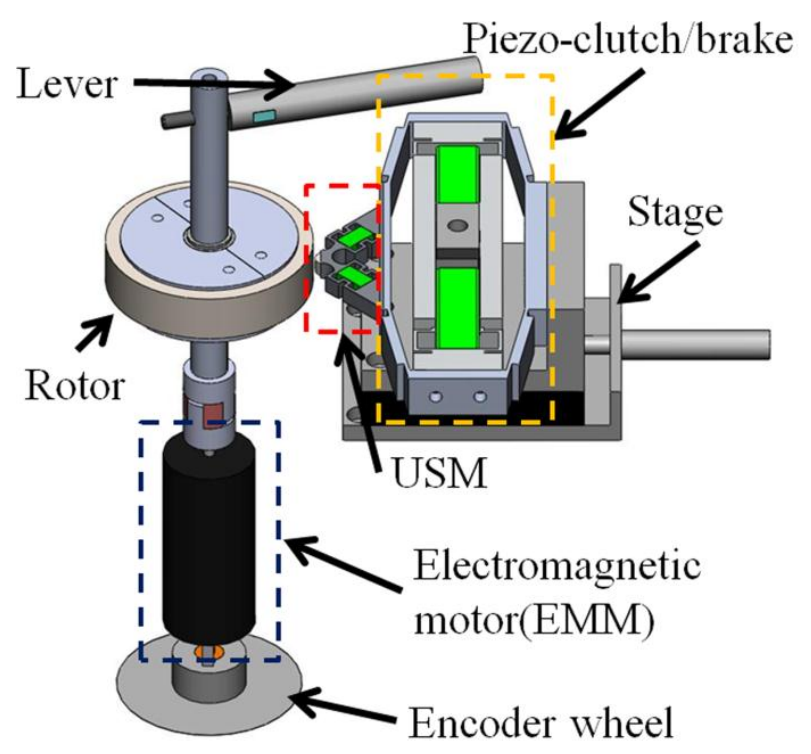

(a)

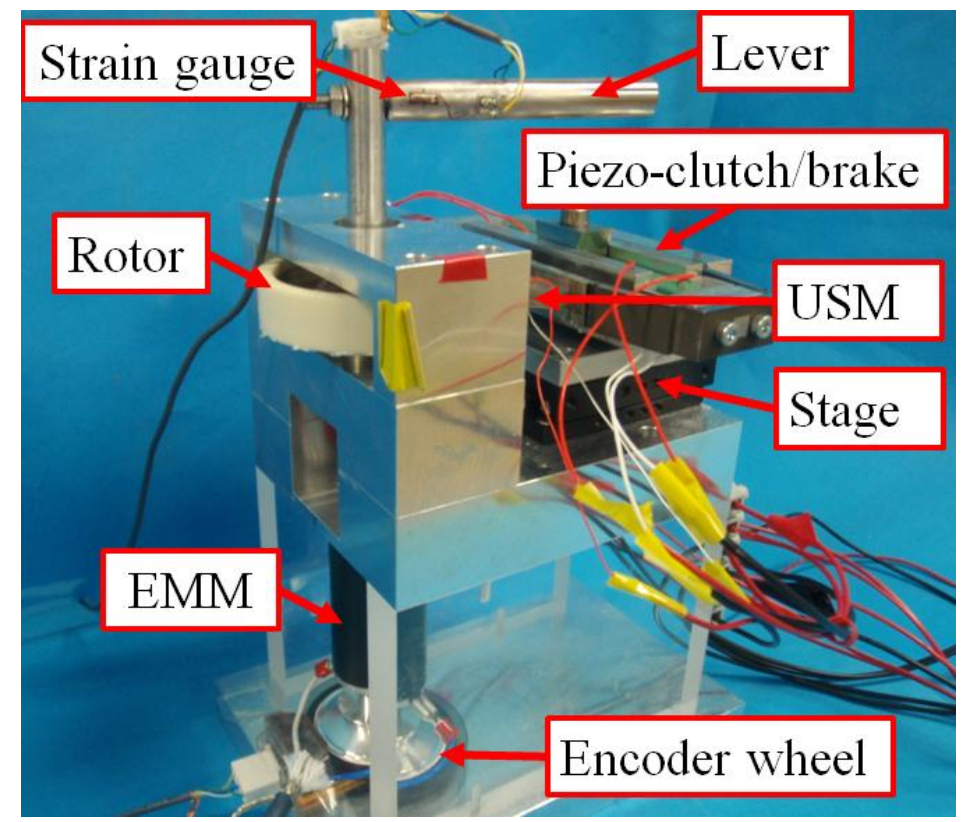

(b)

Fig. 1 Hybrid actuator system: (a) combined system, (b) photograph of constructed device.

\subsection{Ultrasonic motor}

The stator of the USM is set on the piezo-clutch, as shown in Fig. 1(a). This type of USM has a simple design compared with other types of USMs [15]. Two MPAs (NEC-Tokin, AE0505D08) are arranged perpendicular to each other in a holder $[16,17]$. An alumina semicylinder contact tip was adhered on the head of USM and an alumina ring covered the rotor. The friction coefficient between them was approximately 0.06 . When unipolar sinusoidal voltages with a phase difference of $90^{\circ}$ are applied to each MPA, the head of the stator vibrates with an elliptical displacement, as shown in Fig. 2. The rotor rotates due to friction when the head is in contact with the rotor. In general, a USM offers the advantages of a fast response and the existence of a self-holding force when the electric power supply is cut off. The MPA used in the USM has the time response of approximately $0.01 \mathrm{~ms}$ and can be driven up to about $45 \mathrm{kHz}$ [18]. Hence the stator can generate a torque by about $0.03 \mathrm{~ms}$ even at a rough estimate in theory. However, under an actual operating condition that applied voltage was $40 \mathrm{~V}_{\mathrm{p}-\mathrm{p}}$ at $23.5 \mathrm{kHz}$ and preload was $20 \mathrm{~N}$, the measured response time of a USM was measured at $8 \mathrm{~ms}$ for generating torque of $160 \mathrm{mNm}$. This value contained the operation time of a sensor system and the 
delay caused by compliances of shaft and lever. It is hard to directly measure the accurate time response of torque the stator generates.

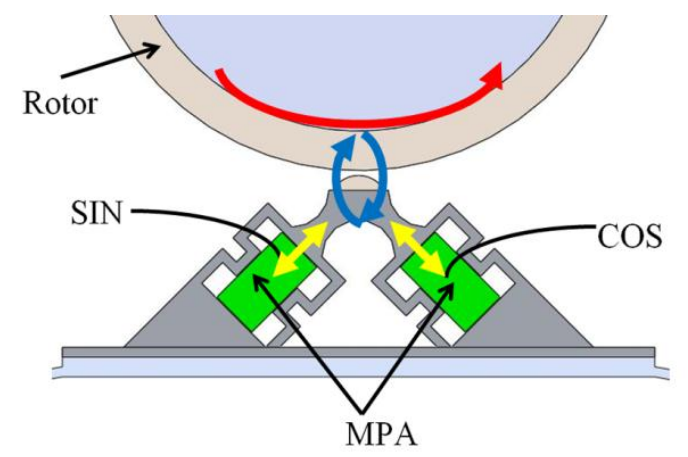

Fig. 2 Operating principle of V-shaped USM.

\subsection{Piezoelectric clutch}

Figure 3 and Table 1 show the operating principle and performance of the piezo-clutch. It contains four MPAs (NEC-Tokin AE1010D16) that can be expanded outward by applying a DC voltage. Generally, a preload is applied between the head of the USM and the rotor, as shown in Fig. 3(a). When the MPAs are expanded, both ends of the mechanical amplifier are pushed so that the head of the USM moves downward and separates from the rotor, as shown in Fig. 3(b). When the electric power supply to the USM is cut off, the clutch operates as a brake. Since the MPAs have a fast response as mentioned before, the contact and separation between the rotor and head of the USM can be performed at high speeds. The piezo-clutch required $1.2 \mathrm{~ms}$ to obtain the maximum deformation without a load. In an actual operation, the reaction time becomes shorter because a smaller deformation is enough to control the friction between the rotor and the head. Hence, the piezo-clutch has a rapid response and is useful for force-feedback compared to existing clutches such as an electromagnetic clutch which has the reaction time of at least $10 \mathrm{~ms}$.

Table 1 Performance of piezo-clutch

\begin{tabular}{|l|c|}
\hline Max. displacement $[\mu \mathrm{m}]$ & 257 \\
\hline Max. contact force $[\mathrm{N}]$ & 50.6 \\
\hline Max. static friction torque $[\mathrm{mNm}]$ & 300 \\
\hline Reaction time for max. deformation $[\mathrm{ms}]$ & 1.2 \\
\hline
\end{tabular}

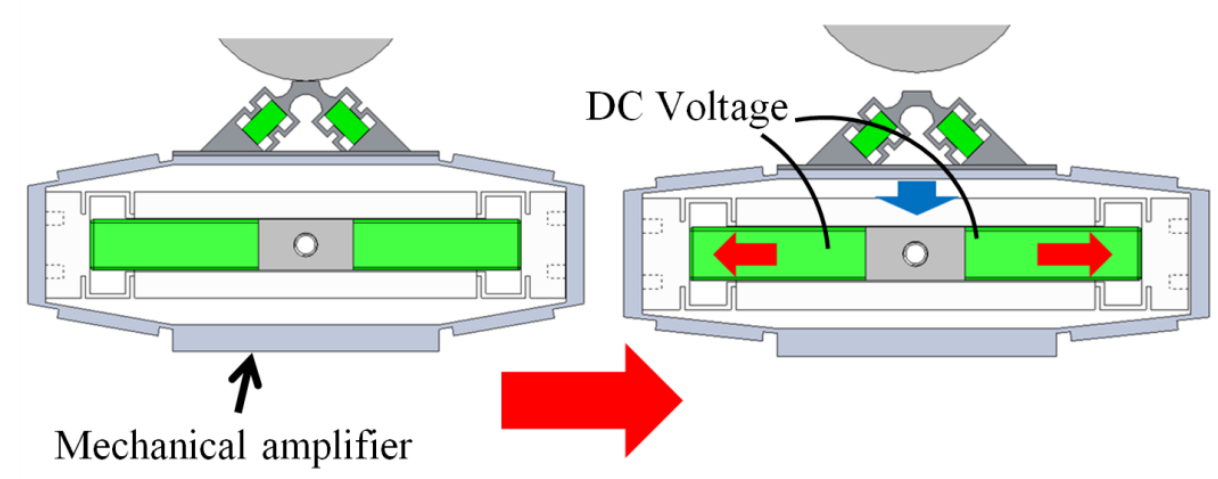

Applied DC Voltage

(a)

(b)

Fig. 3 Operating principle of piezo-clutch: (a) clutch ON, (b) clutch OFF. 
The coreless DC motor (Maxon, RE25) used in the AS has excellent response characteristics compared to other types of an EMM due to small inertia of an armature and small inductance. From the specifications of this motor provided by the manufacture, the electrical and mechanical time constants are about $0.1 \mathrm{~ms}$ and $4.2 \mathrm{~ms}$, respectively [19]. Hence the time constant of torque can be estimated at about $0.1 \mathrm{~ms}$. This value indicates the EMM has good performance, however, the USM is still superior to the EMM in torque response. In this system, the EMM is connected directly to the rotor of the USM, as shown in Fig. 1(a). Therefore, torque control is easy, but a large torque cannot be generated.

\section{Force-feedback display system}

Figure 4 shows the scene of use of a single degree-of-freedom force-feedback display system. In this system, the user pinches the lever, and virtual feelings are controlled by operating the lever. The rotation of the lever not only turns a virtual object in the display but also gives the object a linear motion in any direction according to situations. However, one kind of motion per object can be selected because of a single degree-of-freedom motion. For example, to represent the sense of a sawing, the pinched lever is turned clockwise or counterclockwise corresponding to pulling or pushing a saw. The saw in the display moves right and left.

Figure 5 show a diagram of the force-feedback display system in this study. The rotation angle and torque are measured by an optical encoder and strain gauge on the lever, respectively. These measured data are imported to a computer program developed in MATLAB/Simulink (Mathworks, Inc.) through a data acquisition (DAQ) board. The program behavior for the force-feedback is shown in Fig. 5(b). In this program, the control method was changed by represented feeling. In the case of Fig. 4, the brake is controlled at each setting interval by controller. Then, a signal is generated to control the actuators. Therefore, the force feedback is represented by the piezo-clutch, the EMM, and the USM, which are controlled by a signal from the program. In addition, a virtual object that has some patterns of force feedback is shown on the display. The behavior of this object is synchronized with the lever operation, as mentioned above.

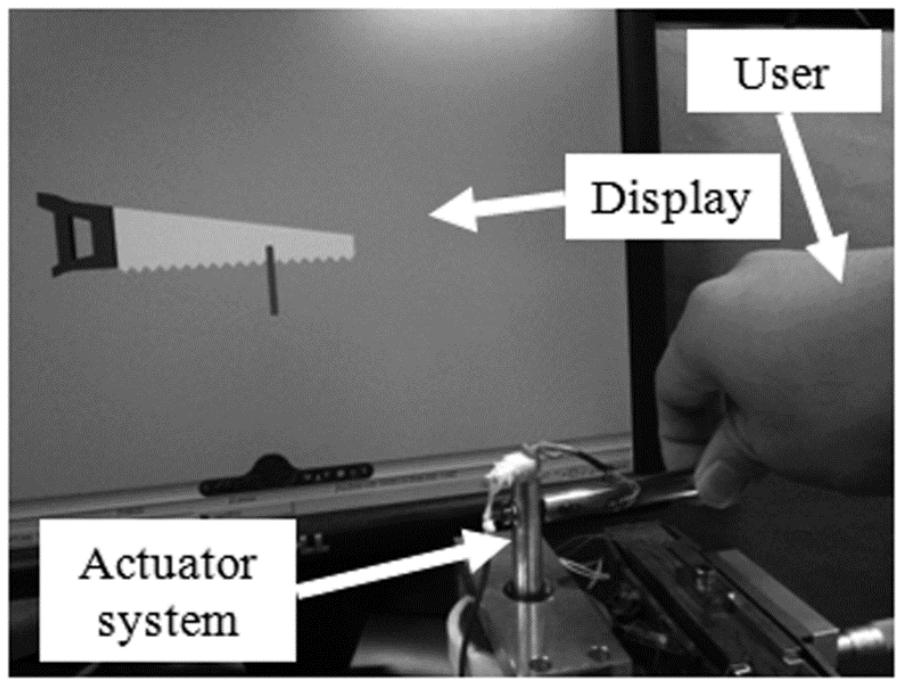

Fig. 4 The scene of use of force-feedback display system. 


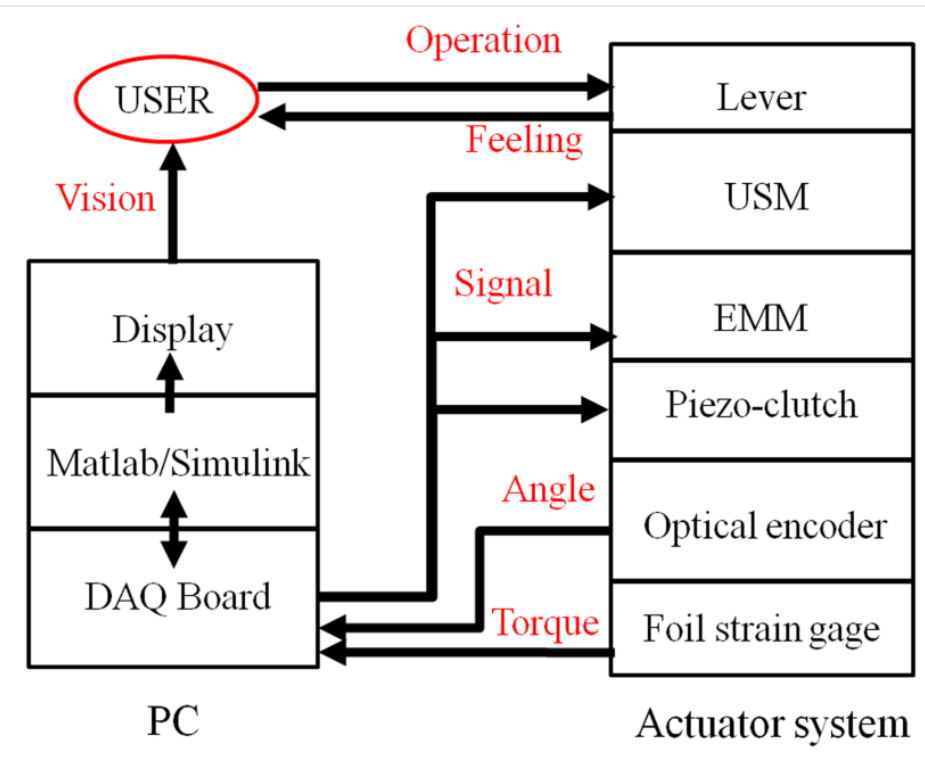

(a)

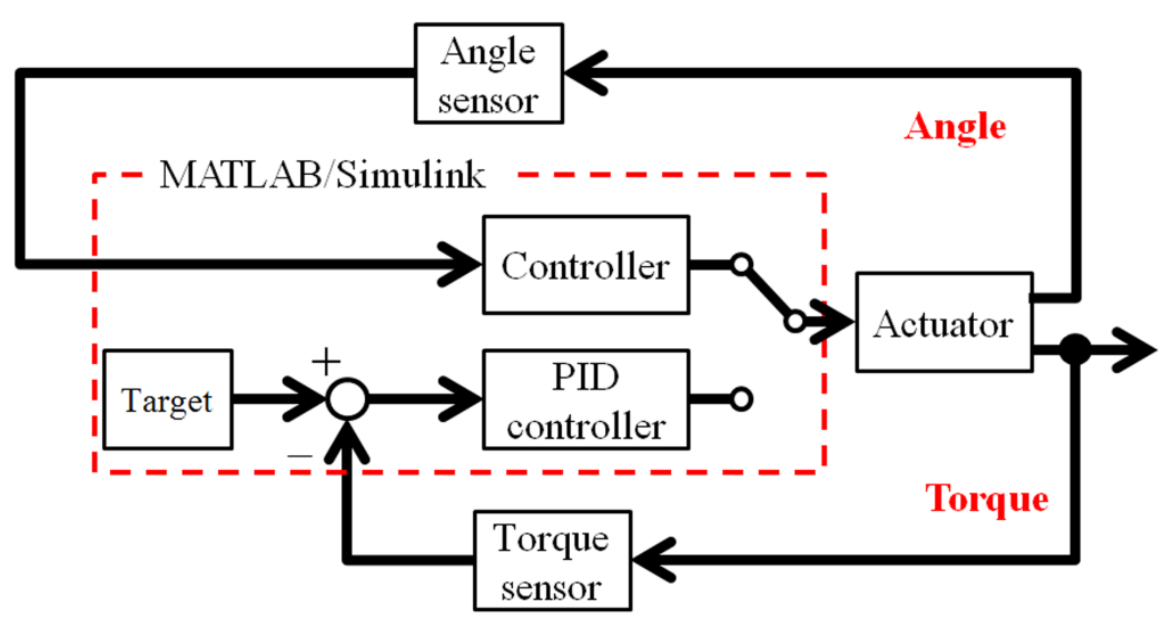

(b)

Fig. 5 Diagram of force-feedback display system: (a) overview of force-feedback display system and

(b) block diagram of force-feedback program in Matlab/Simulink.

4. Experiment of force-feedback display

\subsection{Diagram of force feedback}

The force-feedback display using the hybrid actuator system was evaluated. In this study, the roughness was defined as a discontinuous reaction force. On the other hand the elastic was defined as a continuous reaction force. The represented feelings and combinations of actuators are shown in Table 3. In this experiment, three types of feelings are represented: roughness, friction, and elasticity. The roughness was represented by on-off control at defined positions. The friction reaction was represented by PID control of the piezo-clutch. The elasticity was represented by two methods: one is the use of the EMM and piezo-clutch, and the other is a combination of the USM and piezo-clutch. The aim of this comparison is to clarify whether the USM as well as the EMM can represent the elastic reaction or not. Supposing the USM can do it, the EMM will not be necessary. 
Table 2 Combination method of force-feedback display

\begin{tabular}{|c|c|c|c|}
\hline Haptic situation & USM & EMM & Clutch/Brake \\
\hline Roughness & & & $\circ$ \\
\hline Friction & & & $\circ$ (as Brake) \\
\hline \multirow{2}{*}{ Elasticity } & & $\circ$ & $\circ$ \\
\cline { 2 - 4 } & $\circ$ & & $\circ$ (as Brake) \\
\hline
\end{tabular}

\subsection{Representation of roughness}

A virtual object with five projections was set as illustrated in Fig. 6. The feeling of a finger stroking the tops of the projections was represented under the following conditions. In this experiment, the virtual finger size was considered as zero. The width of each projection was set to a rotation of $1 \mathrm{deg}$, and the gap of each projection was also set to $1 \mathrm{deg}$. Only the piezo-clutch was used for the force-feedback display. Therefore, the user can get the feeling of collisions and free for every $1 \mathrm{deg}$. The measured results for the represented feelings are shown in Fig. 7. These graphs chart the reaction force, clutch voltage, and rotation angle over time. The representation can be divided into three sections: A, B, and C.

In section A, a torque-free state existed. In this section, a voltage of $150 \mathrm{~V}$ was applied to the piezo-clutch. The user did not feel the reaction force and operated the lever freely because the head of the USM was separated from the rotor.

When the lever angle became $0 \mathrm{deg}$, the state of force feedback changed to section $\mathrm{B}$. In this section, the feeling of tracing the five projections was represented by the piezo-clutch. An impulsive force on the rotor was generated by rapidly switching the clutch voltage, which caused states of both contact and separation between the rotor and the head of the USM.

When the lever angle reached $-9 \mathrm{deg}$, the torque-free state was represented again in section $\mathrm{C}$.

As a result, the switching of collision and free could be represented each $1 \mathrm{deg}$. Therefore, the authors were able to feel the roughness represented by the piezo-clutch. The degree of the represented feeling changed by applying voltage to the piezo-clutch or interval of brake.

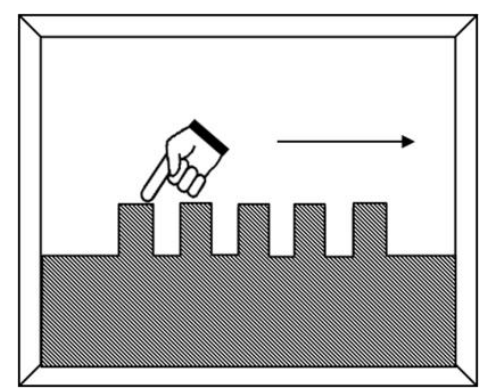

Fig. 6 Virtual object for roughness comb-like shape on the display. 


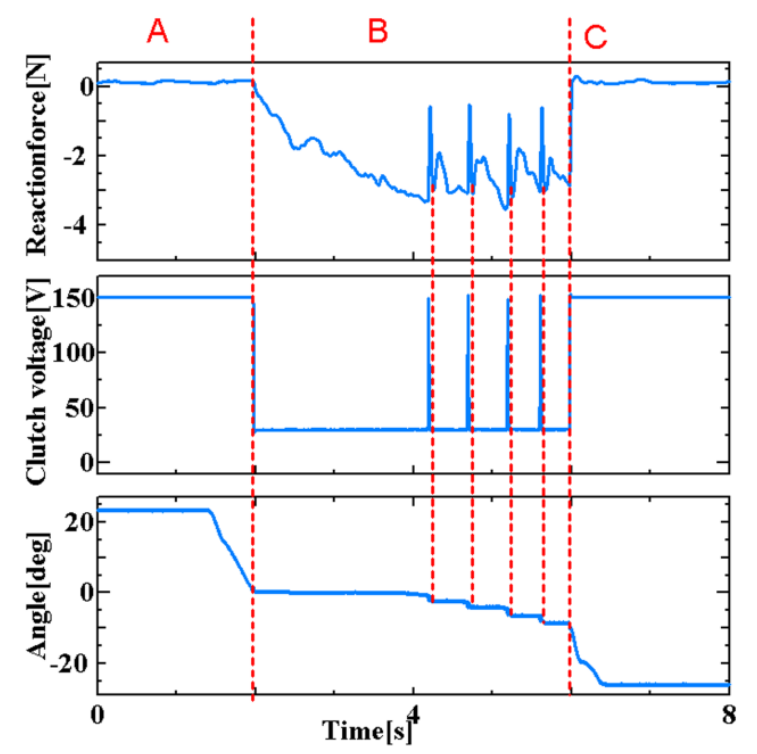

Fig. 7 Charts of reaction force, clutch voltage, and angle for roughness representation.

\subsection{Representation of mass movement with friction}

A block on a flat floor was modeled as a virtual object, as shown is Fig. 8. In this model, the feeling of pushing the block was represented by a friction force controlled by the piezo-clutch. The experimental results for this situation are shown in Fig. 9. This representation can be divided into five sections.

In section A, a torque-free state was represented. In this section, a voltage of $150 \mathrm{~V}$ was applied to the piezo-clutch. The user did not feel a reaction force because the head of the USM was separated from the rotor.

When the lever angle became $0 \mathrm{deg}$, the state of force feedback changed to section $\mathrm{B} 1$. In this section, a feeling of static friction force was represented by the piezo-clutch of which the voltage was kept constant and decided the maximum static friction force. Then, the force-feedback state was shifted to the kinetic friction state in section B2. In section B2, the state of moving block was represented by constant friction force. The reaction force was adjusted by PID control of the applied voltage to the piezo-clutch. The reaction force was defined as

$$
\mathrm{f}=\mathrm{m} \frac{\mathrm{d}^{2} \mathrm{x}}{\mathrm{dt}^{2}}+\mu \mathrm{mg}
$$

where $\mathrm{m}$ is the mass of the block, $\mathrm{x}$ is the position, and $\mu$ is the coefficient of friction. In this experiment, the first term of Eq. 1 was omitted in actually because the acceleration of the mass was supposed to be very small, and the friction force was dominant. Small fluctuations of the reaction force and clutch voltage appeared in this section because of the control limit of an uneven friction force which was originated from roughness on the surface of the rotor or stick-slip phenomenon between the stator and the rotor. However, the user could not feel the fluctuations.

When the external force decreased to below the threshold, the force-feedback state changed to section B3. In this section, the represented feeling switched to the static friction feeling again. Then, if the external force increased, the force-feedback state switched back to the state of section B2. If the lever was moved to separate from the block on the display, the force-feedback state changed to section $\mathrm{C}$. In this section, a torque-free state was represented again. As a result, the authors were able to feel the friction was represented by PID control of the friction between the rotor and the head of the USM. 


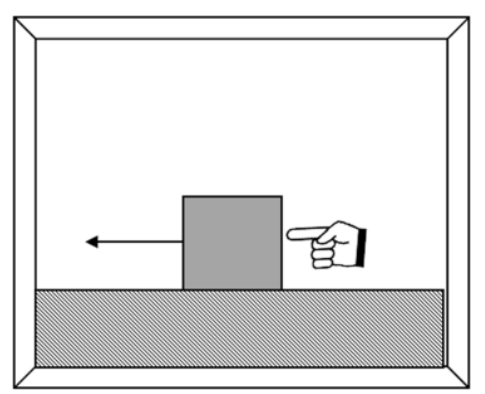

Fig. 8 Virtual block on the display.

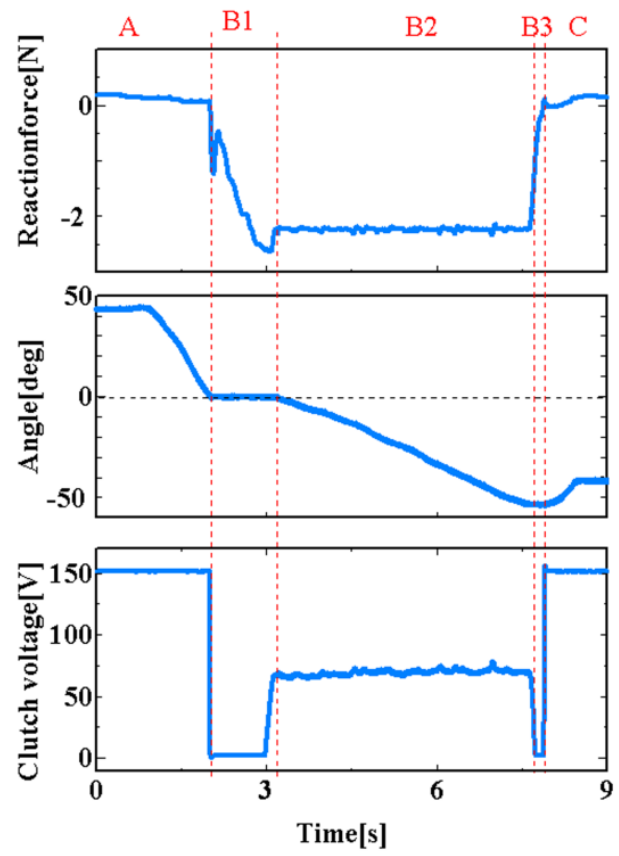

Fig. 9 Charts of reaction force, clutch voltage, and angle in the representation of friction force.

\subsection{Representation of elastic reaction by the USM}

Figure 10 shows a virtual spring set on a floor. In this experiment, the USM and piezo-clutch were used. This haptic model was represented by the following conditions. The expression of the elastic reaction force was set from $0 \mathrm{deg}$ to $-30 \mathrm{deg}$. The reaction force from pushing on the floor was represented at -30 deg, and then, the angle was maintained. The USM, which was driven by a constant applied voltage, was used as a thrust generator, and the piezo-clutch was used for preload adjustment and as a brake.

Figure 11 shows the measured results for the elastic feeling by the USM. This representation can be divided into the following five sections. In the section A, a torque-free state was represented as it was in the models mentioned before. When the lever angle became $0 \mathrm{deg}$, the state of force feedback changed to section B1. In this section, the elastic reaction force was represented by the USM and was defined as

$$
\mathrm{f}=\mathrm{k} x
$$

where $\mathrm{k}$ is the spring constant, and $\mathrm{x}$ is the position. In this experiment, $\mathrm{k}$ was $12.8 \mathrm{~N} / \mathrm{m}$. The reaction force was controlled by the clutch voltage. In this time, applied voltage to the USM was $40 \mathrm{~V}_{\mathrm{p}-\mathrm{p}}$ at $23.5 \mathrm{kHz}$. The relationship of rotation angle, $\theta$, and clutch voltage, $\mathrm{V}$, was defined as

$$
\mathrm{V}=132-0.6 \theta \text { (3). }
$$

The reaction force contained unexpected and uncontrolled variations in sections B1 and B3 while the angle changed smoothly in one direction. Such variations of the reaction force expressed an unwanted roughness. The range of represented elastic feelings was from $0 \mathrm{deg}$ to $-30 \mathrm{deg}$. If the rotation angle became less than $-30 \mathrm{deg}$, the force-feedback state changed to section B2. In this section, the feeling of pushing the floor was represented by holding the rotor with the piezo-brake. At this time, the USM 
was stopped. The movement of the lever was limited by the piezo-brake at the floor. Then, the applied force was decreased below the threshold, and the force-feedback state changed to section B3. In this section, the elastic feeling was represented by the USM. Then, the rotation angle was over 0 deg, and the force-feedback state changed to the torque-free state in section $\mathrm{C}$.

In the representation of the elastic feeling by the USM, a large reaction force was able to be generated rapidly. However, it was not easy to express consecutive and smooth changes in the reaction force because of the non-uniform thrust and the friction force between the head of the USM and the rotor.

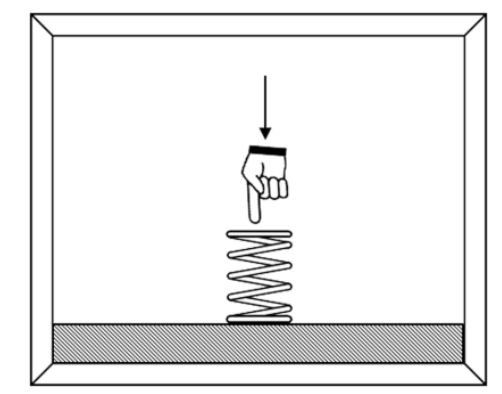

Fig. 10 Virtual spring on the display.

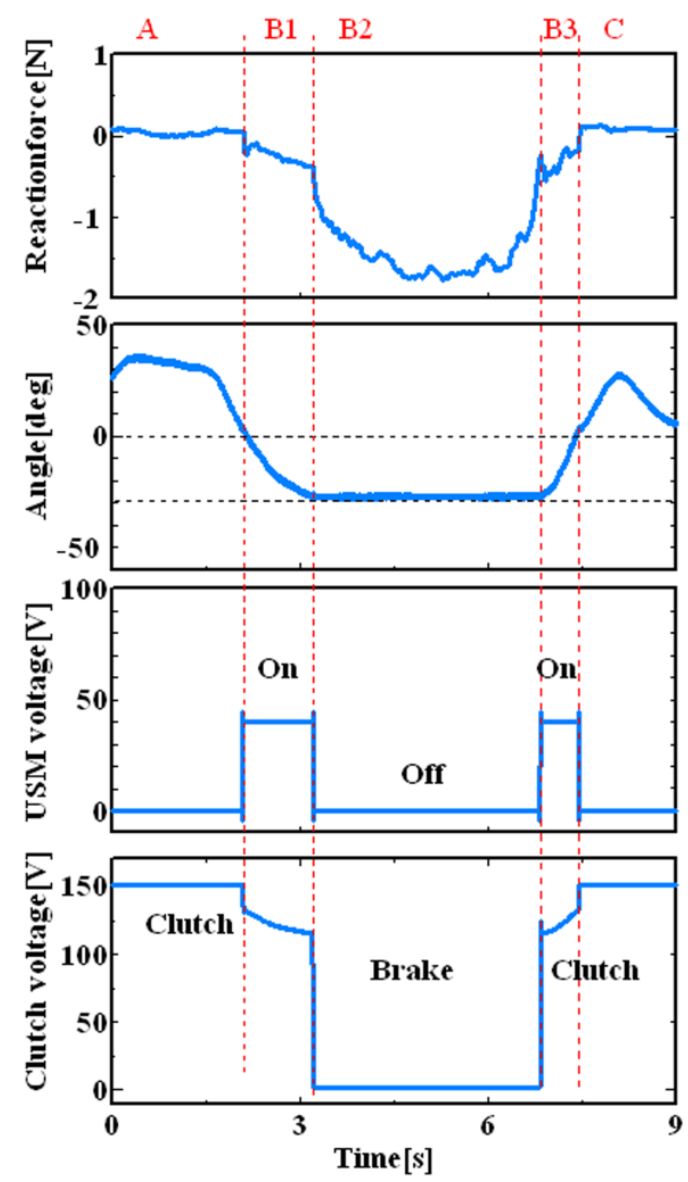

Fig. 11 Charts of reaction force, clutch voltage, and angle in the representation of the elastic reaction force by the USM.

\subsection{Representation of elastic reaction by the EMM}

The EMM and the piezo-clutch were used to represent the elastic reaction. This experiment was performed under the same conditions as the case represented by the USM. The EMM generated thrust, and the piezo-clutch worked as a brake. 
Figure 12 shows the results for the elastic feeling by the EMM. This representation can be divided into the following five sections. Section A had a torque-free state, and the clutch voltage was maintained at $150 \mathrm{~V}$.

When the lever angle became $0 \mathrm{deg}$, the state of force feedback changed to section $\mathrm{B} 1$. In this section, the feeling of the elastic reaction force was represented by the EMM. The reaction force by the EMM changed smoothly unlike the USM. This method is different from the case in which the USM was used. The reaction force was controlled by PWM control of the EMM as a function of the rotation angle. The range of represented elastic feelings was from $0 \mathrm{deg}$ to $-30 \mathrm{deg}$. The relationship of the reaction force and duty ratio was defined as $0.02 \mathrm{~N} / \%$. If the rotation angle became less than $-30 \mathrm{deg}$, the force-feedback state changed to section B2. In this section, the feeling of pushing the floor was represented by holding the rotor with the piezo-brake. The movement of the lever was limited by the piezo-brake at the floor. Then, the external force was decreased to below the threshold of the angle, and the force-feedback state changed to section B3. In this section, the elastic feeling was represented by the EMM again. Then, the rotation angle became over $0 \mathrm{deg}$, and the force-feedback state changed to the torque-free state in section $\mathrm{C}$.

As a result, the authors were able to feel the elasticity represented by the EMM and had an impression that the feeling by the EMM was better than that produced by the USM because the change in the reaction force was smooth. In this model, the piezo-brake was used only to represent the reaction force from the floor.

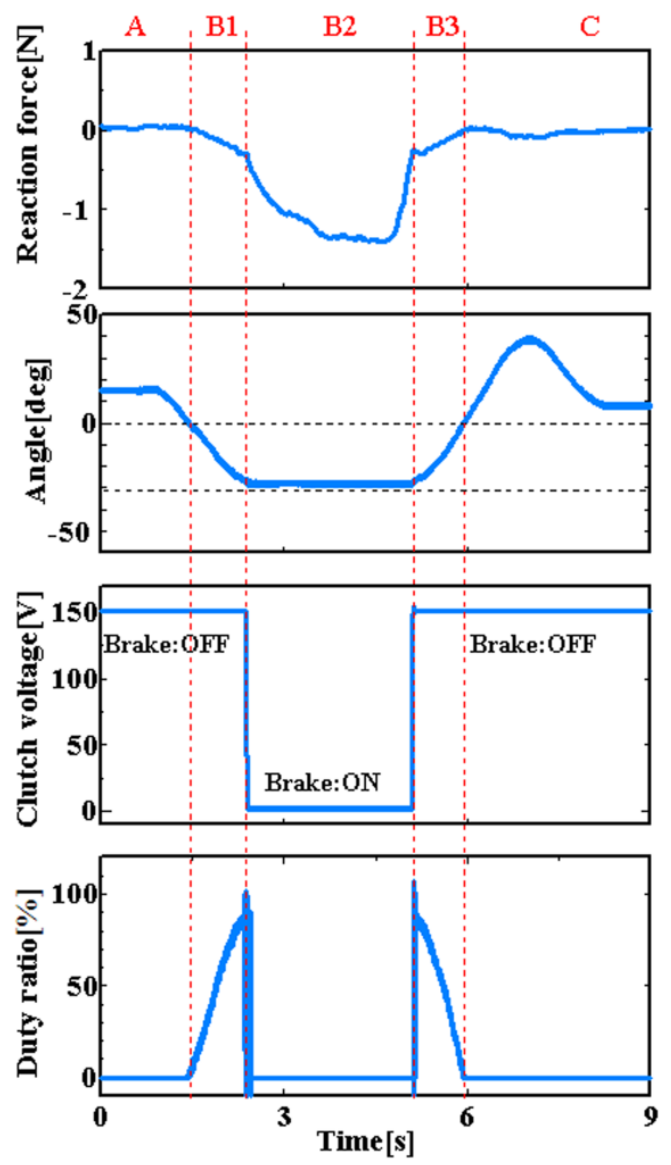

Fig. 12 Charts of reaction force, clutch voltage, and angle in the representation of the elastic reaction force by the EMM.

\section{Summary}

A hybrid actuator system that included a USM, a piezo-clutch/brake, and an EMM was successfully developed. As author's impression, the hardness feeling was realistically represented by the piezo-brake. The elastic feeling was represented by either the EMM or the USM. However, the 
USM was unsuitable for the representation of a contiguous elastic reaction force. The reaction force of the USM was changeable because of the uneven frictional condition on the contact surface. On the other hand, the USM is suitable for generating an impact force like the representation of collision roughness.

In the next stage of this study, a multi-degree-of-freedom system will be developed for more realistic operation. A control method to stabilize the thrust will be realized in order to build a real-time force-feedback system. To evaluate the effectiveness of the force feedback, the reaction force data produced from real objects will be profiled and compared with force-feedback data from the proposed AS. Then, an appropriate control method for force feedback will be considered to approximate the profiled data. A psychological test is also helpful for the evaluation of this system.

Acknowledgment

A part of this work was supported by the Japan Society for the Promotion of Science through a Grant-in-Aid for Scientific Research (B) (21360106).

\section{References}

[1] Information on http://www.sensable.com/

[2] S. Tachi, K. Komoriya, K. Sawada, T. Nishiyama, T. Itoko, M., Kobayashi, and K. Inoue: Adv. Robotics 17 (2003) 199.

[3] Amir M. Tahmasebi, Keyvan Hashtrudi-Zaad and David Thompson Purang Aboimaesumi, IEEE Trans. inf. Technol. Biomed 12 (5) (2008) 658-666.

[4] Kostas Vlachos, Evangelos Papadopoulos and Dionissios N. Mitropoulos, Design and Implementation of a Haptic Device for Training in Urological Operations, IEEE Trans. Rob. Autom. 19 (5) (2003) 801-809.

[5] Siriwan Suebnukarn, Nattharat Phatthanasathiankul, Sunantha Sombatweroje, Phattanapon Rhienmora and Peter Haddawy, Process and outcome measures of expert/novice performance on a haptic virtual reality system, J Dent. 37 (2009) 658-665.

[6] Ursula Buck, Silvio Naether, Marcel Braun and Michael Thali, Haptics in forensics: The possibilities and advantages in using the haptic device for reconstruction approaches in forensic science, Forensic Sci. Int. 180 (2008) 86-92.

[7] Uros Mali and Marko Munih, HIFE-Haptic Interface for Finger Exercise, IEEE/ASME Trans. Mechatron 11 (1) (2006) 93-102.

[8] Haruhisa Kawasaki and Tetsuya Mouri, Design and Control of Five-Fingered Haptic Interface Opposite to Human Hand, IEEE Trans. Robot. 23 (5) (2007) 909-918.

[9] Pablo García-Robledo, Javier Ortego, Manuel Ferre, Jorge Barrio and Miguel A. Sánchez-Urán, Segmentation of Bimanual Virtual Object Manipulation Tasks Using Multifinger Haptic Interfaces, IEEE Trans. Instrum. Meas. 60 (1) (2011) 69-80.

[10] M. Minsky, M. Ouh-Young, O. Steele, F. P. Brooks, and M. Behensky: Feeling and Seeing Issues in Force Display, Computer Graphics (ACM), vol.24,no.2., 1990, pp.235-243.

[11] J. Edward Colgate and J. Michael Brown, Factors affecting the z-width of a haptic display, In Proceedings of the IEEE International Conference on Robotics and Automation, 1994, pp. 3205-3210.

[12] K. Akahane, S. Hasegawa, Y. Koike and M. Sato, The High Definition Haptic Rensering by the Update Frequency of 10kHz, Transactions of the Virtual Reality Society of Japan, vol/9 No.3, 2004, pp.217-226. [in Japanese]

[13] M. Aoyagi, T. Tomikawa, and T. Takano, Proc. 2004 IEEE Ultrasonics Symp. (2004) 899.

[14] T. Takemura, M.Aoyagi, T.Takano, H.Tamura, and Y. Tomikawa, Hybrid Ultrasonic Actuator for Force-Feedback Interface, Jpn. J. Appl. Phys. 47 (5) (2008) 4265-4270.

[15] S. Ueha and Y. Tomikawa, Ultrasonic Motor-Theory and Applications, Clarendon Press, Oxford, U.K., 1993. 
[16] A. Endo, N. Sasaki, and Y. Tomikawa: In 1988 Natl. Conv. Rec., Institute of Electrical Engineers of Japan, No. 733, pp. 899-900 [in Japanese].

[17] K. Mori, T. Kumagae, and H. Hirai, Proc. IEEE 1989 Ultrasonics Symp. (1989) p. 3.

[18] NEC/TOKIN, Multilayer Piezoelectric Actuators, vol.6, 2011.

[19] Maxon motor catalog, May 2012, p77. 